\title{
Gradient-based Texture Cartoon Decomposition
}

\author{
Chuong T. Nguyen and Joseph P. Havlicek \\ School of Electrical and Computer Engineering \\ University of Oklahoma, OK, USA \\ chuong@ou.edu,joebob@ou.edu
}

\begin{abstract}
We proposed an automatic nonlinear texturecartoon decomposition based on the frequency behavior of texture and cartoon across different scales. We measured the ratio of gradient magnitude across modulation domain components and used this ratio to classify the texture and cartoon pixels. The algorithm computed the modulation domain component where texture and cartoon are separated. Our simulation results showed that the proposed algorithm is able to extract meaningful texture and cartoon components from images efficiently.
\end{abstract}

Keywords-cartoon, texture, AM-FM, modulation domain

\section{INTRODUCTION}

We consider the problem of decomposing an image into a structural component and textural component. The structural portion, which is referred to as cartoon, carries broad information about an image and is usually piece-wise smooth curves. The texture component, subsequently referred to as texture, describes oscillating patterns of image textures and noise [1], [2]. For example, when we look at a striped Tshirt, the cartoon consists of lines in the borders of the shirt and the texture are the stripes within the shirt.

A successful texture-cartoon decomposition can lead to improvements for subsequent image processing operations such as compression, edge detection, and image inpainting. For instance, higher overall compression gain can be obtained by decomposing the image into different types of signals and designing optimized encoders to compress these type of signals separately [3]. The cartoon-texture decomposition can eliminate extraneous edges that can partially due to noise or unimportant objects [1], [2]. Such decomposition can retain important edges in image denoising [4].

The texture-cartoon decomposition is, however, an illposed problem. As texture and cartoon are loosely defined, image features can be considered as texture in one scale, but they can be cartoon at another scale. For example, when we look at a tree at a far distance, leaves can be classified as textures. However, we can consider these leaves as cartoon at a closer viewing distance. In addition, human judgement can also play an important role in deciding whether an object is part of texture or not.

Most works in the texture-cartoon decomposition are in the partial differential equation (PDE) setting. The texture and cartoon are modeled to lie in different functional spaces.
The solution is found by solving a convex regularized optimization problem [4]-[7]. The quality of texture and cartoon decomposition depends on signal models used to describe them and the regularization parameter. Despite approaches to find suitable values for the regularization parameter [7], [8], the cartoon edges often bleed into the texture components.

Meyer, Averbuch and Coifman [3] proposed an image compression scheme where an image is decomposed into multi-layered components such as texture and cartoon. The authors used a suitable basis for each each layer of signal in order to increase compression gain. Stark, Elad, and Donoho drew ideas from [3] and [6] to create a hybrid approach that used total variation regularization and basis matching. They designed two dictionaries, each of which contains basis functions that are tuned for either cartoon or texture. The texture and cartoon were subsequently extracted by projecting the image onto these basis functions.

Apart from the PDE and basis representation approaches, Buades et al. [1] proposed a nonlinear texture-cartoon decomposition. They observed that the total variation of texture and cartoon features behave differently before and after a lowpass filtering. A weight assignment scheme were then used to classify texture and cartoon features. While the algorithm produced good texture and cartoon separation, the results depended on the bandwidth parameter of the lowpass filter.

In this paper, inspired by the work of Buades et al. [1], we proposed an automatic nonlinear texture-cartoon decomposition algorithm. In particular, we measured the ratio of gradient magnitude across modulation domain components and used this ratio to determine the component where the change between cartoon and texture of a pixel is most likely to occur. Once the component is determined, we used a hard threshold strategy to classify texture and cartoon pixels to obtain a weight matrix. The texture component is then obtained by multiplying the original image with the weight matrix. The cartoon is the difference between the original image and the texture component.

\section{BACKGROUND}

Let $f: \mathbb{R}^{2} \rightarrow \mathbb{R}$ be a continuous image. Let $u: \mathbb{R}^{2} \rightarrow \mathbb{R}$ be the cartoon component. Let $v: \mathbb{R}^{2} \rightarrow \mathbb{R}$ be the texture component. The cartoon-texture decomposition aims 
to extract $u$ and $v$ as

$$
f=u+v .
$$

\section{A. Total variation regularization approaches}

Most of the the texture-cartoon decomposition approaches are formulated in the partial differential equation setting, we will give a short description of the underlying models. Readers can refer to [4], [6], [7] for deeper analysis.

Rudin, Osher, and Fatemi [4] solved (1) in the context of a denoising problem. They assumed that the cartoon $u$ belongs to a class of bounded variation (BV) functions and the texture $v$ is a finite energy function. Both $u$ and $v$ are solved simultaneously in the convex minimization setup

$$
\underset{u \in \mathrm{BV}, v \in \mathrm{L}^{2}}{\arg \min }\left(\int|D u|+\lambda\|v\|_{\mathrm{L}^{2}}^{2}\right),
$$

where $\lambda$ is a positive tuning parameter and the integral of $D u$ measures the total variation of signal $u$. The computed texture $v$, however, contains cartoon edges. Meyer [5] provided an alternative model for the texture component in (2). Instead of being $\mathrm{L}^{1}$ or $\mathrm{L}^{2}, v$ belongs to functions in a Banach space featured by a $G$-norm model which allows features to have high oscillation but can still retain low energy norm. Many successful texture-cartoon decomposition algorithms have been derived from the Meyer formulation, e.g., [6], [7].

\section{B. Traditional filtering approaches}

1) The Linearized Meyer Model: Buades et al. [1] observed that a linearized version of the original Meyer model is indeed the classical highpass-lowpass filtering problem. Let $K_{\sigma}$ be a lowpass filter; the texture-cartoon decomposition problem can be viewed as a problem of designing a suitable lowpass filter $\mathrm{K}_{\sigma}$ to capture $u$ and $v$ as

$$
\begin{aligned}
u & =K_{\sigma} * f \\
v & =f-K_{\sigma} * f,
\end{aligned}
$$

where $\sigma$ is the scale parameter that determines the filter bandwidth and $*$ denotes the convolution operator.

Intuitively, the bandwidth parameter $\sigma$ controls the amount of high frequency features that will be retained. Therefore, this model can not separate the texture and cartoon features when their frequencies are overlapped.

2) Nonlinear texture-cartoon classification: Buades et al. [1] observed that the local total variation (TV) of texture features and cartoon features behave differently when filtered by a lowpass filter $K_{\sigma}$. The ratio of local TV before and after the lowpass filter $K_{\sigma}$ is applied tends to be lower in the texture region than that in the cartoon region. Based on this observation, the authors used a nonlinear mapping similar to soft-thresholding to classify pixels into the two categories.

Even though the decomposition algorithm does not compute solutions that converge to those of the TV regularization approaches [1], it produces good quality texture-cartoon separation with a non-iterative implementation. The solutions of this method, however, depend on the selection of the bandwidth $\sigma$ of the lowpass filter $K_{\sigma}$. Without a properly tuned $\sigma$, the solutions can change drastically.

\section{TEXTURE-CARTOON DECOMPOSITION}

We represent the image $f$ as a sum of $K$ non-stationary amplitude modulation (AM) functions and frequency modulation (FM) functions

$$
f=\sum_{k=1}^{K} f_{k}=\sum_{k=1}^{K} a_{k} \cos \left(\varphi_{k}\right)
$$

where $a_{k}: \mathbb{R}^{2} \rightarrow \mathbb{R}^{+}$is the AM function and $\varphi_{k}: \mathbb{R}^{2} \rightarrow \mathbb{R}$ is the phase modulation function [9]. Both $a_{k}$ and $\varphi_{k}$ are assumed to be locally smooth. The FM functions are given by the gradient of $\varphi_{k}$, i.e., $\nabla \varphi_{k}=\left[\varphi_{k x} \varphi_{k y}\right]^{T}$, where the second subscript denotes partial differentiation. The discrete $\mathrm{AM}$ and FM functions are computed using the demodulation algorithm in [10]. We arranged the $K$ AM-FM components in ascending order based on the magnitude of the FM vector in (4), i.e., $f_{1}$ carries low-frequency components and $f_{K}$ contains high-frequency components.

The key ingredient the cartoon-texture separation in [1] as well as in this paper lies in the computation of image gradient. For 1D AM-FM signal representation, the derivative of component $f_{k}$ is obtained as

$$
f_{k}^{\prime}=a_{k}^{\prime} \cos \left(\varphi_{k}\right)-\varphi_{k}^{\prime} a_{k} \sin \left(\varphi_{k}\right) .
$$

We performed an approximation to (5) to make it more robust to noise. Since the AM function $a_{k}$ is locally smooth, we estimated the $1 \mathrm{D}$ derivative of $f_{k}$ in (5) as

$$
f_{k}^{\prime} \approx-\varphi_{k}^{\prime} a_{k} \sin \left(\varphi_{k}\right)
$$

Extended the $1 \mathrm{D}$ derivative in (6) to $2 \mathrm{D}$, we computed a metric $T_{\ell}$ to quantify the gradient magnitude of the first $\ell$ AM-FM components according to

$$
T_{\ell} \approx \sqrt{\left(\sum_{k=1}^{\ell} \varphi_{k x} a_{k} \sin \left(\varphi_{k}\right)\right)^{2}+\left(\sum_{k=1}^{\ell} \varphi_{k y} a_{k} \sin \left(\varphi_{k}\right)\right)^{2}}
$$

In (7), $T_{1}$ is the approximated gradient magnitude of the lowest frequency component while $T_{K}$ is the approximated gradient magnitude of the image $f$.

Similar to Buades et al. [1], we defined the gradient magnitude ratio at every pixel as

$$
D_{\ell}=\frac{T_{K}-T_{\ell}}{T_{K}}
$$

where $1 \leq \ell \leq K$. At a pixel $(m, n)$ in the image grid, $D_{\ell}(m, n)$ measures the relative difference between the gradient magnitude of the whole image and the gradient 
magnitude of the first $\ell$ components. $D_{\ell}$ is maximum when $\ell=1$ and decreases monotonically towards 0 as $\ell$ increases.

We defined $f_{\beta}$ to be the AM-FM component where the change between texture and cartoon is likely to happen at each pixel. We first created a mask $M$ such that $M(m, n)=$ $\alpha$ if $D_{\alpha}>0.25$ and $M(m, n)=0$ otherwise. The index $\beta$ is then estimated according to

$$
\beta=\operatorname{median}(M) .
$$

Finally, we applied a hard threshold strategy to create a weight matrix $w$ where a weight of one means texture and a weight of zero means cartoon

$$
w= \begin{cases}1, & \text { if } T_{\beta} \geq 0.25 \\ 0, & \text { if } T_{\beta}<0.25\end{cases}
$$

The cartoon and texture are then computed as

$$
\begin{aligned}
& u=w \cdot f, \\
& v=f-u .
\end{aligned}
$$

\section{Simulation Results}

We ran the proposed algorithm on the Kodak image dataset and standard test images. The results are shown in Fig. 2. For each test image, the texture-cartoon decomposition results are demonstrated by row. The original image is in the left column, the cartoon component $u$ is in the middle column, and the texture component $u$ is on the right. Fig. 2(b) and Fig. 2(c) show the cartoon and the texture component of the image kodim05. We can see that the overall structure of the image is retained in the cartoon, while the fine textures in the roofs and windows are extracted into the texture. Fig. 2(e) and Fig. 2(f) depict the cartoon and the texture component of the fingerprint image. The algorithm is able to extract most of repeating curves in the original image and put into the texture component. The cartoon contains mostly low-frequency residual. Fig. 2(h) and Fig. 2(i) illustrates the cartoon and the texture component of the Lena image. Oscillating patterns in her pant, shirt, and in the table are successfully extracted to the texture. The edges in her hands and table are still kept in the overall structure of the image.

Figure. 1 illustrates the texture and cartoon obtained from the linearized Meyer model discussed in Sec. II-B1 and the Buades et al. [1]. Notice that the linearized Meyer model includes strong edges in the texture component in Fig. 1(e). We also see the variation of results by the Buades et al. [1] in Fig. 1(b),(c),(f),(g). These decomposition results vary according to the selection of the filter bandwidth $\sigma$. The results of the proposed method are shown in Fig. 1(d) and (h). The proposed algorithm is able to separate the texture and cartoon automatically.

\section{CONCLUSION}

We proposed an automatic nonlinear texture-cartoon decomposition based on the frequency behavior of texture and cartoon across different scales. We measured the ratio of gradient magnitude across modulation domain components and use this ratio to classify the texture and cartoon pixels. Our simulation results demonstrated that the proposed algorithm is able to extract texture and cartoon components from images efficiently. While this work followed a similar path as Buades et al. [1], our results do not depend on the lowpass filter bandwidth which is critical to the separation process. Currently, we set the threshold parameter in the hard threshold process empirically to 0.25 . We are experimenting with machine learning techniques to overcome this limitation.

\section{REFERENCES}

[1] A. Buades, T. Le, J.-M. Morel, and L. Vese, "Fast cartoon + texture image filters," IEEE Trans. Image Proc., vol. 19, no. 8, pp. 1978-1986, Aug. 2010.

[2] J.-L. Starck, M. Elad, and D. L. Donoho, "Image decomposition via the combination of sparse representations and a variational approach," IEEE Trans. Imag. Proc., vol. 14, no. 10 , pp. $1570-1582$, Oct. 2005.

[3] F. G. Meyer, A. Z. Averbuch, and R. R. Coifman, "Multilayered image representation: Application to image compression," IEEE Trans. Image Proc., vol. 11, no. 9, pp. 10721080, Sept. 2002.

[4] L. Rudin, S. Osher, and E. Fatemi, "Nonlinear total variation based noise removal algorithms," Physica D, vol. 60, pp. 259268, 1992.

[5] M. Meyer, Oscillating patterns in image processing and nonlinear evolution equations: The fifteenth Dean Jacqueline B. Lewis memorial lectures. AMS, 2001.

[6] L. Vese and S. Osher, "Modeling textures with total variation minimization and oscillating patterns in image processing," $J$. Sci. Comput., vol. 19, pp. 553-577, Oct. 2003.

[7] J. Aujol, G. Gilboa, T. Chan, and S. Osher, "Structure-texture image decomposition - Modeling, algorithms and parameter selection," Intl. J. Comput. Vis, vol. 67, no. 1, pp. 111-136, 2006.

[8] R. Shahidi and C. Moloney, "Decorrelating the structure and texture components of a variational decomposition model," IEEE Trans. Image Proc., vol. 18, no. 2, pp. 299-309, Feb. 2009.

[9] J. P. Havlicek, D. S. Harding, and A. C. Bovik, "Multidimensional quasi-eigenfunction approximations and multicomponent AM-FM models," IEEE Trans. Image Proc., vol. 9, no. 2, pp. 227-242, Feb. 2000 .

[10] C. T. Nguyen, R. A. Sivley, and J. P. Havlicek, "First results in perceptually-based AM-FM image filtering," in Proc. IEEE Southwest Symp. Image Anal., Interp., Santa Fe, NM, Mar. 24-26, 2008, pp. 77-80. 

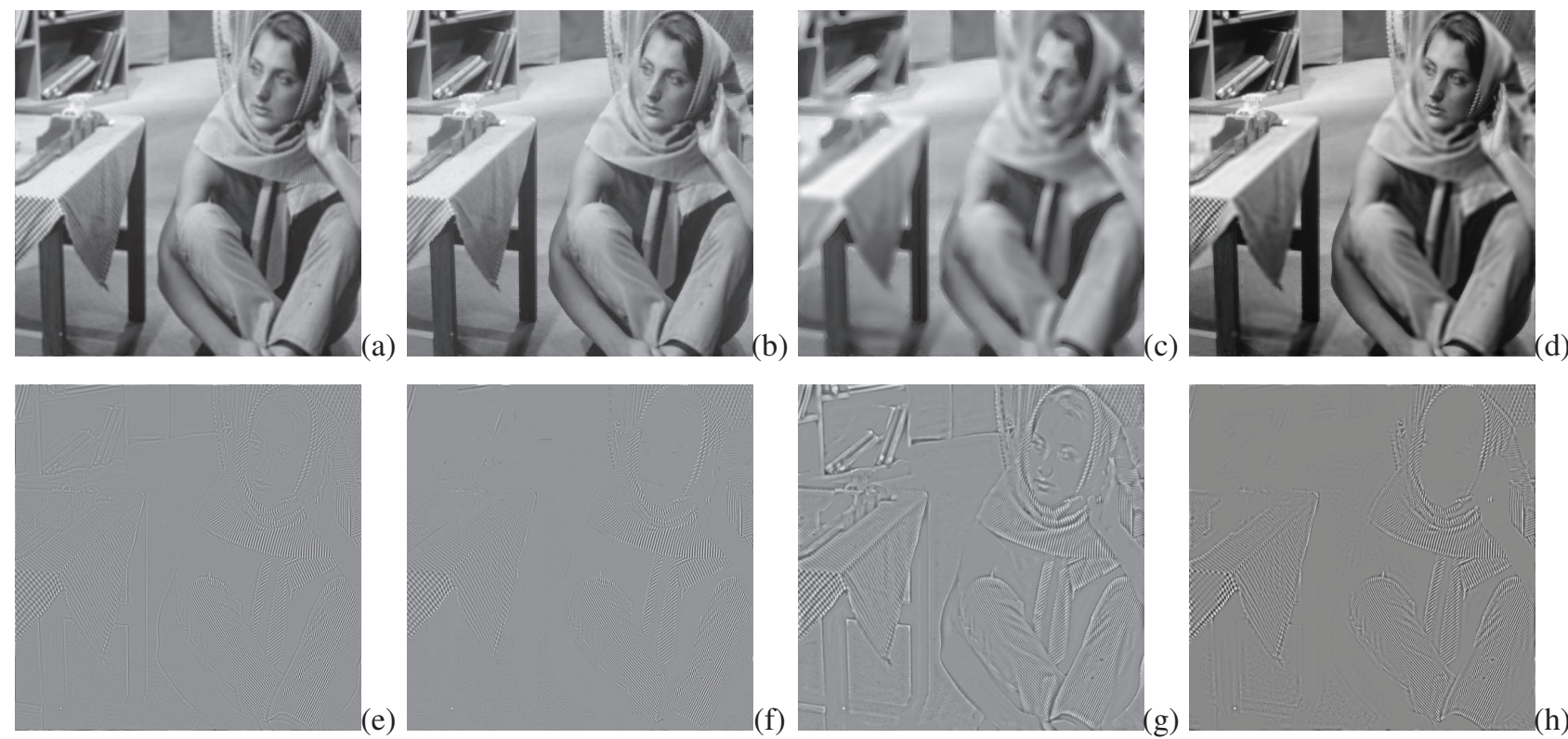

Figure 1. Texture Cartoon Decomposition Examples of Barbara. (a) Cartoon obtained by linearized Meyer model. (b) Cartoon obtained by Buades et al. [1] with $\sigma=1.0$. (c) Cartoon obtained by Buades et al. [1] with $\sigma=3.0$. (d) Cartoon otained by our method. (e) Texture obtained by linearized Meyer model. (f) Texture obtained by Buades et al. [1] with $\sigma=1.0$. (g) Texture obtained by Buades et al. [1] with $\sigma=3.0$. (h) Texture obtained by our method.
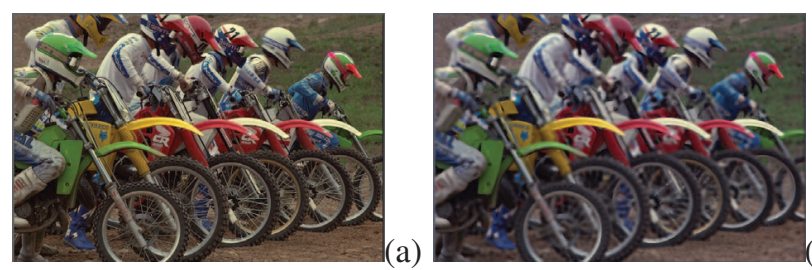

(b)
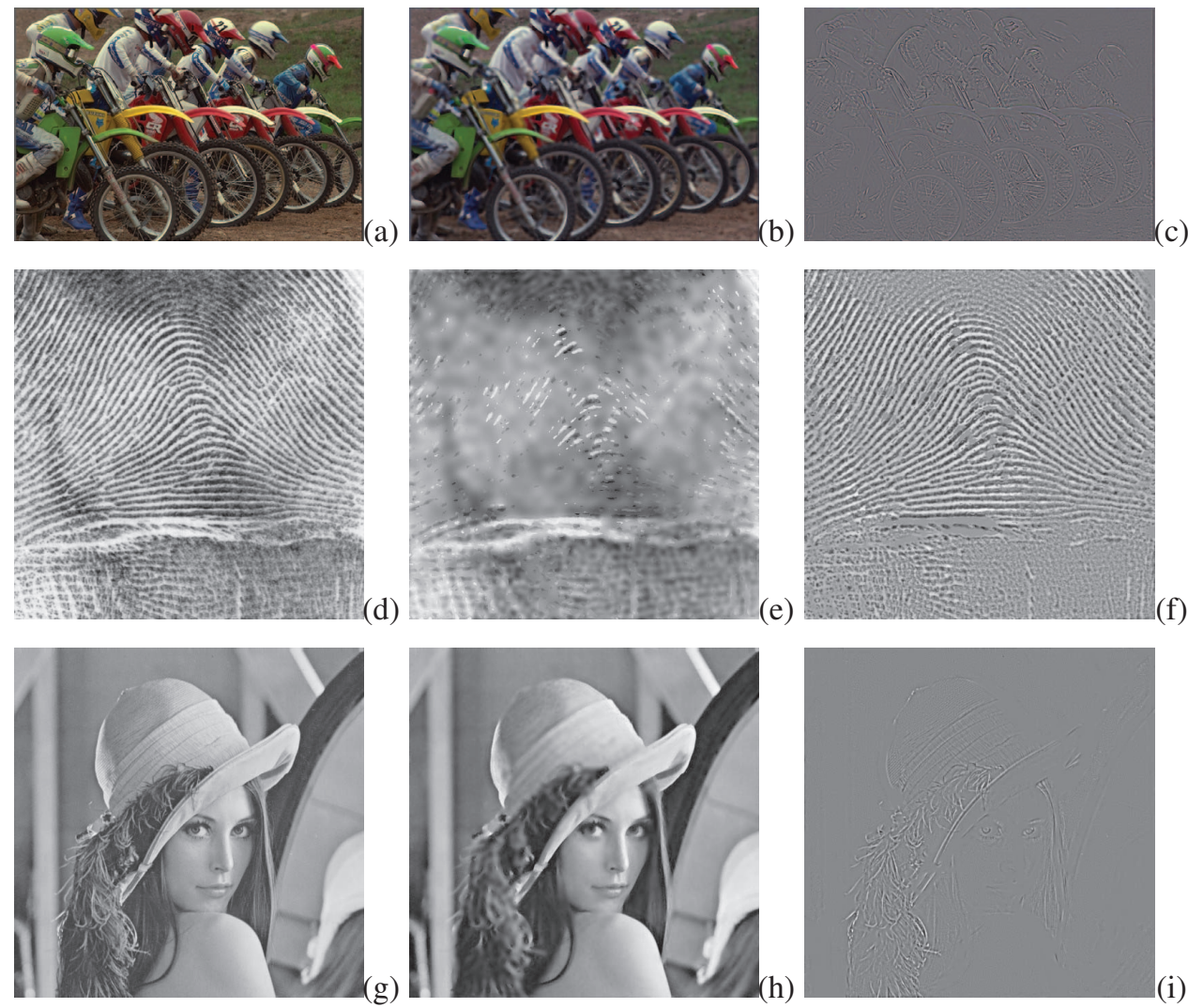

f)

Figure 2. Texture Cartoon Decomposition Examples. (a) Original kodim05 from Kodak. (b) Cartoon component of (a). (c) Texture component of (a). (d) Original fingerprint. (e) Cartoon component of (d). (f) Texture component of (d). (g) Original Lena. (h) Cartoon component of (g). (i) Texture component of $(\mathrm{g})$. 